2 Enhanced Disrupting Effect of Benzophenone-1

3 Chlorination Byproducts to Androgen Receptor: Cell-based

4 Assays and Gaussian Accelerated Molecular Dynamics

\title{
5 Simulations
}

6 Tingjie Zhan ${ }^{\dagger}$, Shixuan Cui ${ }^{\dagger}$, Xujun Liu ${ }^{\dagger}$, Chunlong Zhang ${ }^{\#}$, Yu-ming M. Huang ${ }^{\ddagger}$,

7 Shulin Zhuang ${ }^{\dagger}$, *

8

$9 \dagger$ Key Laboratory of Environment Remediation and Ecological Health, Ministry of

10 Education, College of Environmental and Resource Sciences, Zhejiang University,

11 Hangzhou 310058, China.

$¥$ Department of Physics and Astronomy, Wayne State University, Detroit, MI 48201, 13 USA.

\# Department of Environmental Sciences, University of Houston-Clear Lake, TX 77058, USA.

* Correspondence: shulin@zju.edu.cn (S. Zhuang); ymhuang@wayne.edu (YM

19 Huang). 
Text S1 Chlorination process of BP-1. For the investigation of time-course profiles of BP-1 and its chlorinated products, the concentration of BP-1 was set at $10 \mathrm{mg} / \mathrm{L}$ to obtain enough amount of byproducts, and FC was added according to the FC/BP-1 molar ratio of 1:1.5, 1:3, 1:6, 1:15. During the chlorination, the reaction mixture was collected at different time intervals $(0,20 \mathrm{~s}, 40 \mathrm{~s}, 60 \mathrm{~s}, 80 \mathrm{~s}, 100 \mathrm{~s}, 120 \mathrm{~s}, 1 \mathrm{~h}, 2 \mathrm{~h}$ and $14 \mathrm{~h}$ ) for the reaction kinetics and the evaluation of antiandrogenic activity. The residual chlorine was quenched using $100 \mu \mathrm{L}$ sodium thiosulfate solution $(10 \mathrm{~g} / \mathrm{L})$. BP-1 is primarily attacked by $\mathrm{HOCl}$, which is the hydrolysis product of $\mathrm{NaOCl}$ (Eq.1, Eq.2).

$\mathrm{ClO}^{-}+\mathrm{H}_{2} \mathrm{O} \rightleftarrows \mathrm{HOCl}+\mathrm{OH}^{-}$

$\mathrm{HOCl}+\mathrm{BP}-1 \rightarrow$ Chlorinated products

During the chlorination, $10 \mathrm{~mL}$ samples of the reaction mixture were taken from flask at $0,10 \mathrm{~s}, 20 \mathrm{~s}, 40 \mathrm{~s}, 60 \mathrm{~s}, 80 \mathrm{~s}, 100 \mathrm{~s}, 120 \mathrm{~s}, 1 \mathrm{~h}, 2 \mathrm{~h}$, and the residual chlorine was quenched using $100 \mu \mathrm{L}$ sodium thiosulfate solution $(10 \mathrm{~g} / \mathrm{L})$ in a HPLC vial for the reaction kinetics. $10 \mathrm{~mL}$ samples of the reaction mixture were taken from the flask for the extraction by HLB solid-phase microextraction (Waters, USA). The extract was re-dissolved in $200 \mu \mathrm{L}$ methanol for HPLC analysis or re-dissolved in DMSO for toxicity evaluation.

Text S2 UPLC-MSMS. UPLC-MS/MS (Waters Corp., Milford, MA, USA) with the reverse phase Acquity UPLC HSS T3 column $(2.1 \mathrm{~mm} \times 100 \mathrm{~mm}, 1.8 \mu \mathrm{m}$, Waters, Dublin, Ireland) was used to detect BP-1 and the chlorination mixture. The mobile phase contained $0.1 \%$ formic acid-water (A) and methanol (B) at a flow rate of $0.3 \mathrm{~mL} / \mathrm{min}$. The linear gradient program for mobile phase was set as follows: $0 / 5$, 7/100, 8/100, 10.5/5, 11/5 (min/B\%) The UPLC-MS/MS was performed in the 
negative ion mode of electrospray ionization (ESI) source. The detection of BP-1 and its chlorinated products were detected using MS scan mode.

Text S3 UPLC-QTOF-MSMS. The chlorinated products of BP1 were analyzed with an ultra-performance liquid chromatography (UPLC) (Waters Corp., Milford, MA, USA) coupled with an AB Triple TOF $5600+127$ system (AB SCIEX, Framingham, USA). Reaction products were separated by the Agilent ZORBAC SB-C18 column $(2.1 \times 100 \mathrm{~mm}$, particle size: $3.5 \mu \mathrm{m})$ with the column temperature of $35^{\circ} \mathrm{C}$. Water $(0.1 \%$ formic acid (FA) $)$ and acetonitrile $(0.1 \% \mathrm{FA})$ were used as the LC mobile phases with a constant flow rate of $0.6 \mathrm{~mL} / \mathrm{min}$. The gradient for mobile phases was set as: $0 \mathrm{~min}, 95 \%$ water $(0.1 \% \mathrm{FA})$; and hold for $20 \mathrm{~min}$; $20 \mathrm{~min}, 50 \%$ water $(0.1 \%$ FA); $30 \mathrm{~min}, 5 \%$ water $(0.1 \% \mathrm{FA})$ and $31 \mathrm{~min}, 95 \%$ water $(0.1 \% \mathrm{FA})$. The detection wavelength was set at $254 \mathrm{~nm}$ with the maximum allowed error of $\pm 5 \mathrm{ppm}$. The UPLC-Q-TOF-MS/MS was performed in the negative MS mode through APCI source. The collision energy was set at $40 \mathrm{~V}$ with a spread of $\pm 20 \mathrm{~V}$. The $\mathrm{m} / \mathrm{z}$ of precursor ions and product ions of analytes were set within the range of 100-1500 Da and 50-1500 Da, respectively. The calibration of exact mass was carried out automatically by the Automated Calibration Delivery System. The PeakView Software version 1.2 (AB SCIEX, Framingham, USA) was used for the analysis of chlorinated products.

\section{Text S4 Two-hybrid recombinant human AR yeast bioassay. AR antagonistic} activity of BP-1 and the chlorinated products was conducted via the human recombinant AR two-hybrid yeast bioassay following reported protocols. ${ }^{1,2}$ pGBT9 AR plasmid and the yeast cells transformed with pGBT9AR plasmid were provided by Dr. Erik Jan Dubbink (Erasmus University, Holland) and Prof. Zijian Wang (Research Center for Eco-Environmental Sciences, Chinese Academy of Sciences, 
Beijing, China), respectively. The yeast cells were cultured in Synthetic dextrose/ leucine/ tryptamine (SD/-Leu/-Trp medium, catalogue: 4823-6) from MoBiTec GmbH (Goettingen,Germany). Chemicals were mixed with positive control for antagonism assay in yeast cell suspension. To avoid the false positive effect caused by cytotoxicity, the result was excluded if OD600 value was less than $10 \%$ of the negative control. The enzyme reaction was started by adding $o$-nitrophenyl $\beta$-Dgalactopyranoside. The reactions were terminated by the addition of sodium carbonate, and the adsorption was measured at $420 \mathrm{~nm}$. DMSO and DHT (50 nM) were used as negative and positive control, respectively. Finally, the activities of $\beta$ galactosidase were calculated by the reported method. The assay was determined at least in biological triplicate for three technical replicates.

Text S5 System setup of GaMD simulations. The simulation systems, including apo AR LBD and ligand-bound AR LBD, were first minimized to relax hydrogen atoms (500 steps), sidechains (5000 steps), and the entire protein (5000 steps) gradually. Then, the systems were solvated with TIP3P water molecules ${ }^{3}$ around $12 \AA$ to create a rectangular simulation box. The counter ions, five $\mathrm{Cl}^{-}$, were added to neutralize the systems (about 45,000 atoms in total). Another energy minimization of water molecules (1000 steps) and the entire system (5000 steps) were further performed. The minimized systems were gradually heated to $50 \mathrm{~K}, 100 \mathrm{~K}, 150 \mathrm{~K}, 200 \mathrm{~K}, 250 \mathrm{~K}$, and $300 \mathrm{~K}$ for $10 \mathrm{ps}$ at each temperature.

Text S6 Rigid-protein docking. Glide program implemented in Schrodinger Suite $2020^{4,5}$ was used to perform all docking simulations. BP-1, P1, and P2 were docked into $20 \mathrm{AR}$ proteins that include different structural conformations sampled by the above GaMD simulations. Accordingly, in total 60 docking simulations were performed. To correct Lewis structures and to eliminate mistakes of the ligands in 
downstream computational errors, the compounds were first prepared using the LigPrep program with the OPLS2005 force field. ${ }^{6}$ Glide grids were generated for a receptor with the center located at the AF2 binding site. The inner and outer boxes for docking were set to 10 and $30 \AA$, respectively. The XP docking was finally carried out, and ligands with the best score were selected.

Text S7 Induced-fit docking. The induced-fit docking simulations were performed through the Glide program in Schrodinger Suite 2020. ${ }^{4,5,7}$ BP-1, P1, and P2 were docked into the structure of modelled AR LBD provided by Dr. Huixiao Hong (U.S. Food and Drug Administration). We selected the AF2 site of AR LBD as the binding site, which was defined by Asn705, Met745, Phe764, and Thr877. The inner docking box was set to10 $\AA$, and the Glide default was used for the outer box value. The overall AR LBD was rigid during the docking simulations except for the residues within $5 \AA$ of the docking center.

Text S8 Post-GaMD analysis. The CPPTRAJ program implemented in AmberTools 18 ${ }^{8}$ and VMD tool ${ }^{9}$ were used to calculate time courses of atom distances, H-bonds, dihedral angles, root mean square deviation (RMSD), and root mean square fluctuation (RMSF). The g-cluster program in Gromacs2018.4 package ${ }^{10}$ was applied to cluster MD trajectories into 20 clusters based on backbone RMSD of AR LBD. The major conformations from the 20 clusters were used for XP docking.

The binding interaction energies of BP1, P1 and P2 to AR LBD were evaluated by an end-point energy calculation approach, MM-GBSA calculation. ${ }^{11,12}$ We used the structural ensemble obtained from the final 100-ns GaMD simulations to perform the post-energy calculations. The total interaction energy, $\Delta E_{b i n d}$, associated with the binding of $\mathrm{AR}$ and the byproducts can be expressed as the following form:

$\Delta E_{\text {bind }}=\left\langle E_{\text {complex }}\right\rangle-\left\langle E_{\text {protein }}\right\rangle-\left\langle E_{\text {compound }}\right\rangle$ 
123 The bracket $\langle E\rangle$ denotes the average energy calculated from a given GaMD trajectory.

124 Generalized-Born (GB) model ${ }^{13}$ was used to estimate the polar solvation energy. The

125 model, igb $=5$, was assigned in the sander program of Amber18 package. The salt

126 condition was set to $0.15 \mathrm{M}$. The non-polar solvation term was calculated by the solvent

127 accessible surface area (SASA) model. ${ }^{14}$ The changes in average energy on molecular

128 interactions can be decomposed as follows:

$129 \Delta E_{\text {bind }}=\Delta E_{\text {ele }}+\Delta E_{n p}=\Delta U_{\text {ele }}+\Delta U_{V D W}+\Delta W_{G B}+\Delta W_{n p}$

130 where $\Delta E_{\text {ele }}$ and $\Delta E_{n p}$ are the total electrostatic and hydrophobic energy in the sum of

131 the contributions from both the target molecules and solvent, $\Delta U_{\text {ele }}$ and $\Delta U_{V D W}$ are

132 Coulombic and van der Waals (VDW) interactions contributed from the binding

133 between the protein and ligand, and $\Delta W_{G B}$ and $\Delta W_{n p}$ are the solvent free energy from

134 polar and non-polar cavity energy term.

135 
136 Table $\mathbf{S 1}$ The primers for qPCR.

\begin{tabular}{ccc}
\hline $\begin{array}{c}\text { Gene } \\
\text { symbol }\end{array}$ & Forward primer & Reverse primer \\
\hline ACTB & CTGGAACGGTGAAGGTGACA & AAGGGACTTCCTGTAACAATGCA \\
KLK3 & GAGGTCCACACACTGAAGTT & CCTCCTGAAGAATCGATTCCT \\
NKX3.1 & ATGGGTGGGACATGGAAAAG & AGGCTCACTCAGTCCCTTGC \\
\hline
\end{tabular}

137 
138 Table S2 UPLC-QTOF-MSMS parameters of BP-1 and its chlorinated products.

\begin{tabular}{|c|c|c|c|c|}
\hline Compound & $\begin{array}{l}\text { Retention } \\
\text { time (min) }\end{array}$ & $\begin{array}{l}\text { Precursor } \\
(\mathbf{m} / \mathbf{z})\end{array}$ & Formula & $\begin{array}{l}\text { Fragment ions } \\
\qquad(\mathrm{m} / \mathbf{z})\end{array}$ \\
\hline BP-1 & 14.6647 & 213.0567 & $\mathrm{C}_{13} \mathrm{H}_{10} \mathrm{O}_{3}$ & $\begin{array}{l}65.0077,91.0208,135.0084, \\
169.0648,213.0545\end{array}$ \\
\hline $\mathrm{P} 1$ & 16.3881 & 247.0172 & $\mathrm{C}_{13} \mathrm{H}_{9} \mathrm{O}_{3} \mathrm{Cl}$ & $\begin{array}{l}124.9801,155.0497, \\
168.9692,211.0391,247.0158\end{array}$ \\
\hline $\mathrm{P} 1{ }^{\prime}$ & 17.2510 & 247.0177 & $\mathrm{C}_{13} \mathrm{H}_{9} \mathrm{O}_{3} \mathrm{Cl}$ & $\begin{array}{l}124.9804,155.0496, \\
168.9695,211.0394,247.0168\end{array}$ \\
\hline $\mathrm{P} 2$ & 18.8171 & 280.9783 & $\mathrm{C}_{13} \mathrm{H}_{8} \mathrm{O}_{3} \mathrm{Cl}_{2}$ & $\begin{array}{l}122.9639,153.0341, \\
158.9403,202.9304,280.9448\end{array}$ \\
\hline
\end{tabular}

139

140 
141 Table S3 Structures of BP-1 and its chlorinated products".

$\begin{array}{cccc}\text { Compound } & \text { BCF } \\ \begin{array}{c}\text { 2,4- } \\ \text { 3-chlorine-2,4- } \\ \text { (P1', mCl-BP1) }\end{array} \\ \begin{array}{c}\text { 5-chlorine-2,4- } \\ \text { 3,5-dechlorine-2,4- } \\ \text { dihydroxybenzophenone } \\ \text { (P2, mCl-BP1) }\end{array}\end{array}$


144 Table S4 The antiandrogenic activity of the individual BP-1, P1, P2 and the mixture

145 of P1-P2 and BP1-P1-P2. The toxicity unit (TU) with the inhibitory ratio at 50\%

146 (EC50-TU) of mixture was used for the assessment of joint toxicity.

\begin{tabular}{cccc}
\hline Compound & EC50 $(\boldsymbol{\mu M})$ & $\mathbf{9 5 \%} \mathbf{C i}$ & TU in the mixture \\
\hline BP1 & 12.89 & $9.63-23.34$ & - \\
P1 & 6.13 & $5.41-7.03$ & - \\
P2 & 9.30 & $7.94-11.37$ & $0.80<0.82<1.20$ \\
P1-P2 & 6.04 & $4.61-6.20$ & $0.80<1.05<1.20$ \\
BP-1-P1-P2 & 9.06 & $6.68-12.33$ & \\
\hline
\end{tabular}

147

148 
149 Table S5 The predicted $\mathrm{EC}_{50}$ values of the mixture of P1-P2 and BP1-P1-P2 by the

150 CA model.

\begin{tabular}{cccc}
\hline P1\&P2 & EC 50 $(\boldsymbol{\mu M})$ & RPD (\%) & MDR \\
\hline P1-P2 & 6.943 & -14.95 & 1.15 \\
BP1-P1-P2 & 8.327 & $8.05 \%$ & 0.92
\end{tabular}

$151 \mathrm{RPD}=$ the relative percent difference of the predicted $\mathrm{EC}_{50}$ values compared with the

152 observed $\mathrm{EC}_{50}$ values.

$153 \mathrm{MDR}=$ the ratio of predicted $\mathrm{EC}_{50}$ values to the observed $\mathrm{EC}_{50}$ values.

154 
155 Table S6 Main H-bonds formed between BP-1, P1, or P2 and AR LBD.

\begin{tabular}{|c|c|c|c|c|}
\hline Compound & Donor & Acceptor & $\begin{array}{l}\text { Average H-bond } \\
\text { Distance }(\AA)\end{array}$ & Occupancy $(\%)^{a}$ \\
\hline \multirow{3}{*}{ P1 } & $\mathrm{O} 1$ & Thr877:OG & 3.23 & 0.57 \\
\hline & $\mathrm{O} 3$ & Asn705:OD & 1.84 & 96.62 \\
\hline & $\mathrm{O} 3$ & Leu701:O & 3.27 & 0.06 \\
\hline \multirow{3}{*}{ P1' } & $\mathrm{O} 1$ & Thr877:OG & 2.10 & 12.39 \\
\hline & $\mathrm{O} 3$ & Asn705:OD & 1.85 & 91.85 \\
\hline & $\mathrm{O} 3$ & Leu701:O & 3.15 & 7.41 \\
\hline \multirow{3}{*}{$\mathbf{P 2}$} & $\mathrm{O} 1$ & Thr877:OG & $\mathrm{n} / \mathrm{a}$ & 0.00 \\
\hline & $\mathrm{O} 3$ & Asn705:OD & 1.76 & 100.00 \\
\hline & $\mathrm{O} 3$ & Leu701:O & $\mathrm{n} / \mathrm{a}$ & 0.00 \\
\hline \multirow{3}{*}{ BP-1 } & $\mathrm{O} 1$ & Thr877:OG & 2.10 & 15.57 \\
\hline & $\mathrm{O} 3$ & Asn705:OD & 1.83 & 61.88 \\
\hline & O3 & Leu701:O & 3.18 & 15.11 \\
\hline
\end{tabular}

156 a The H-bond occupancy is calculated as the ratio of the number of conformations

157 with hydrogen bonds to the total number of conformations by CPPTRAJ program in $158 \quad$ AmberTools18.

159 

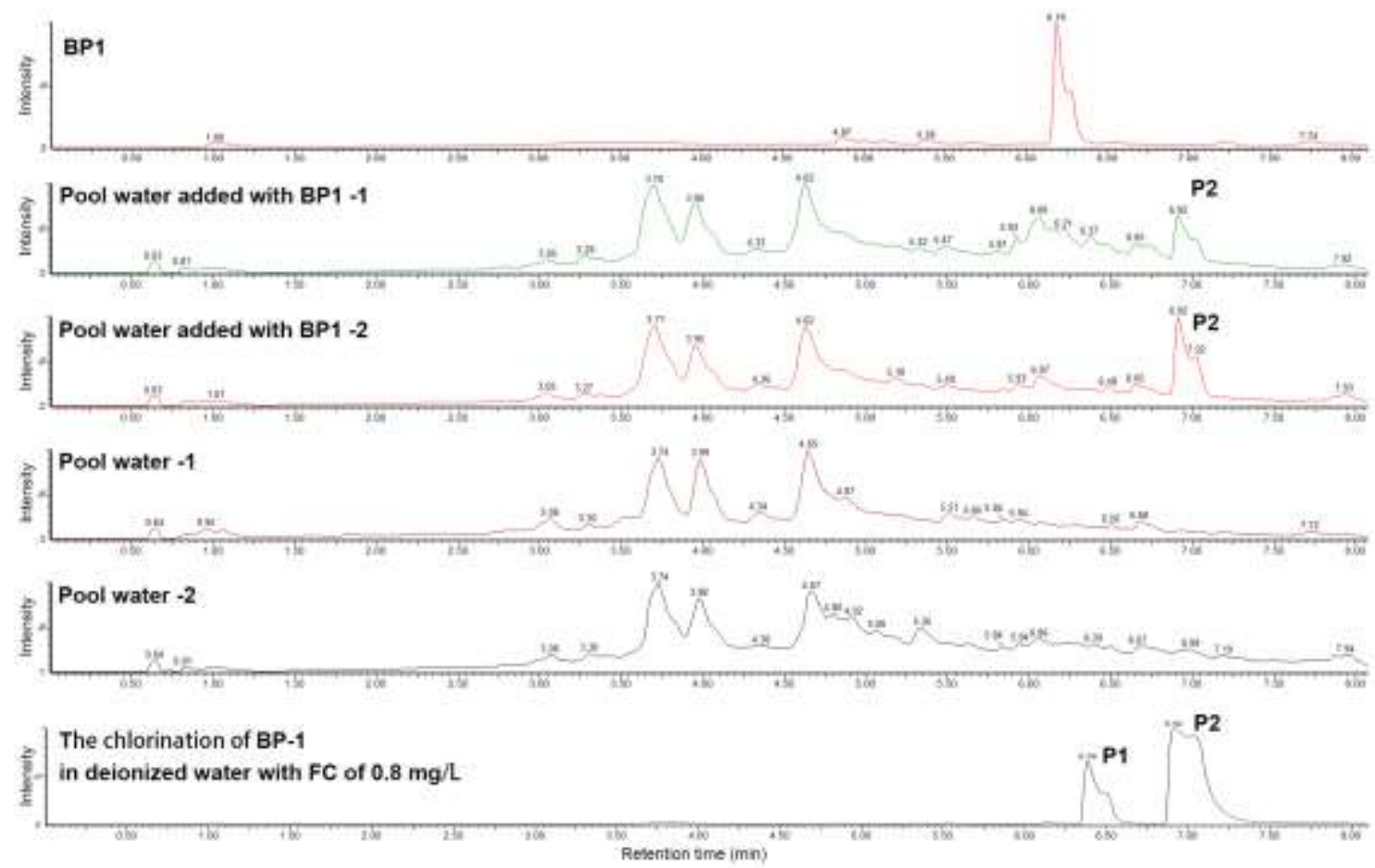

160 Figure S1 The chlorination of BP-1 in swimming pool water or deionized water as

161 measured by UPLC-MSMS. The initial concentration of BP-1 is $20 \mu \mathrm{g} / \mathrm{L}$. The free

162 chlorine with dosage of $0.8 \mathrm{mg} / \mathrm{L}$ was added in deionized water (concentration

163 measured in swimming pool water). The chlorination time in swimming pool water or

164 deionized water was $1 \mathrm{~h}$. The assay was performed in two replicates. 


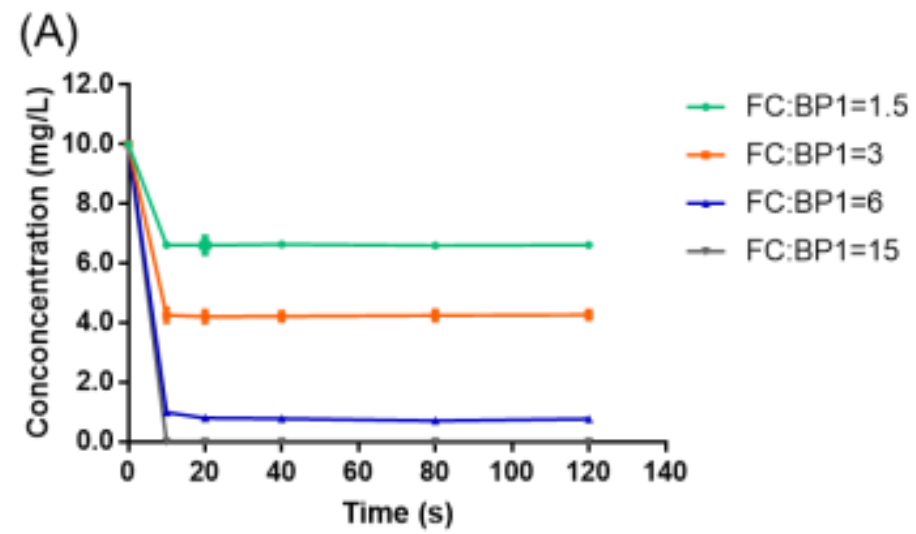

(B)

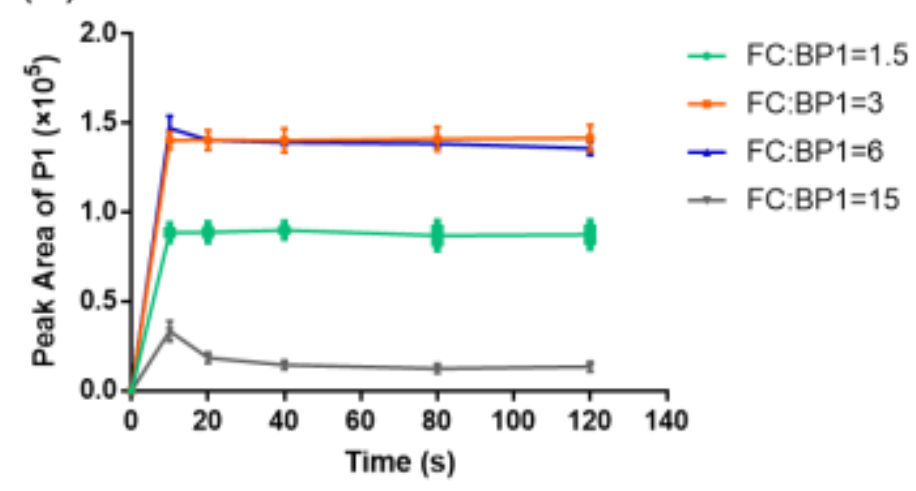

(C)

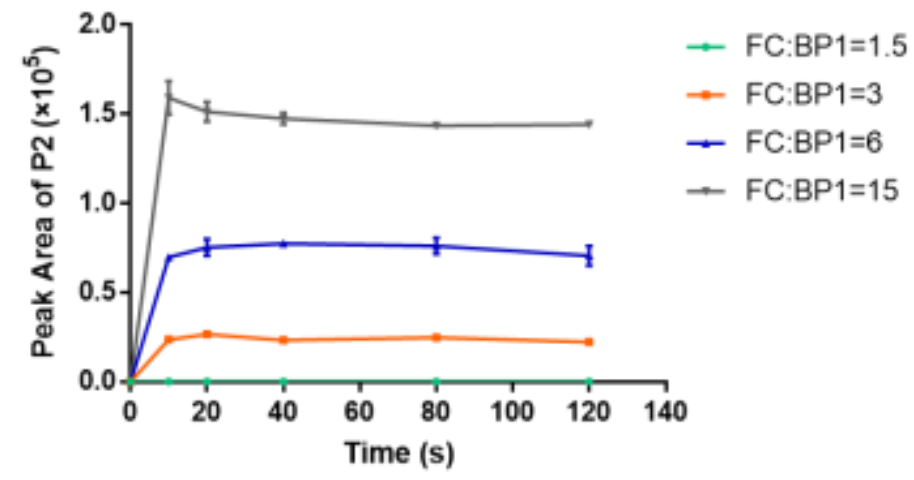

166

167 Figure S2 The time-course profiles of BP-1 (A) and the its major chlorinated products

$168 \mathrm{P} 1(\mathrm{~B})$ and P2 (C) after the treatment with free chlorine at different molar ratios of

169 free chlorine to $\mathrm{BP}-1$ concentration. $\mathrm{pH}=7.0$; temperature: $22 \pm 0.5^{\circ} \mathrm{C}$.

170 

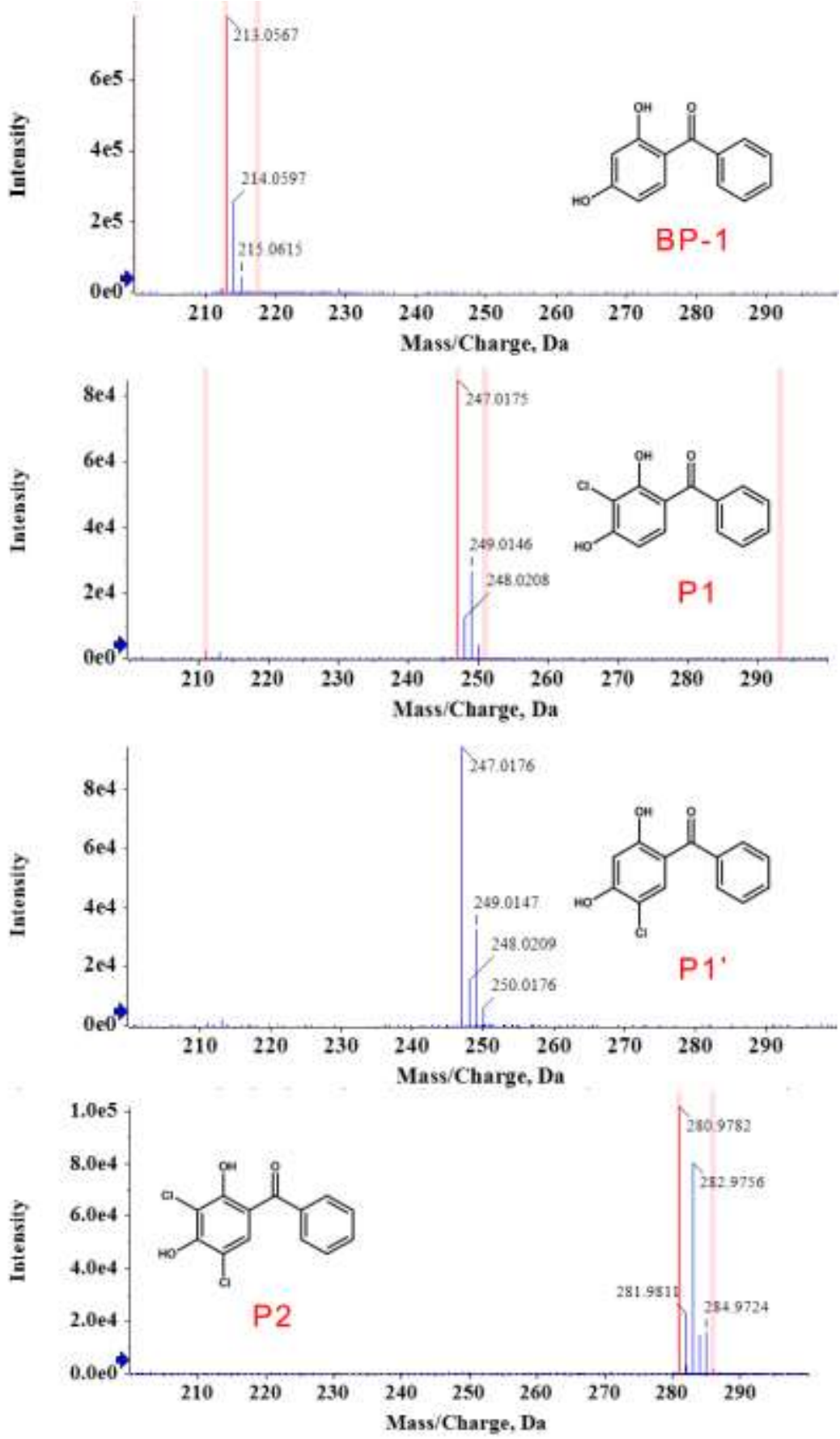

171

172 Figure S3 The QTOF-MS spectrum of BP-1 and three tentatively identified

173 chlorinated products.

174 

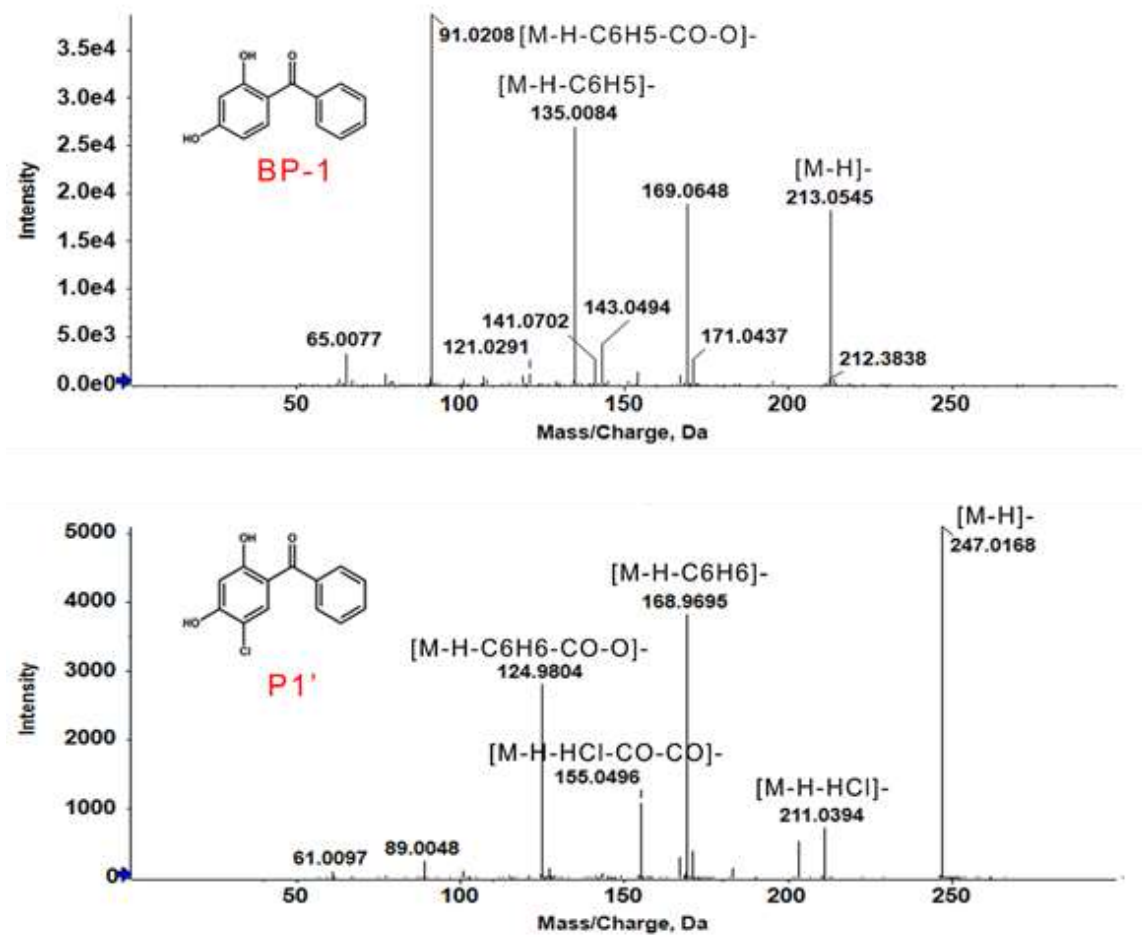

176 Figure $\mathbf{S 4}$ The QTOF-MSMS spectrum of BP-1 and P1'. 
(A)

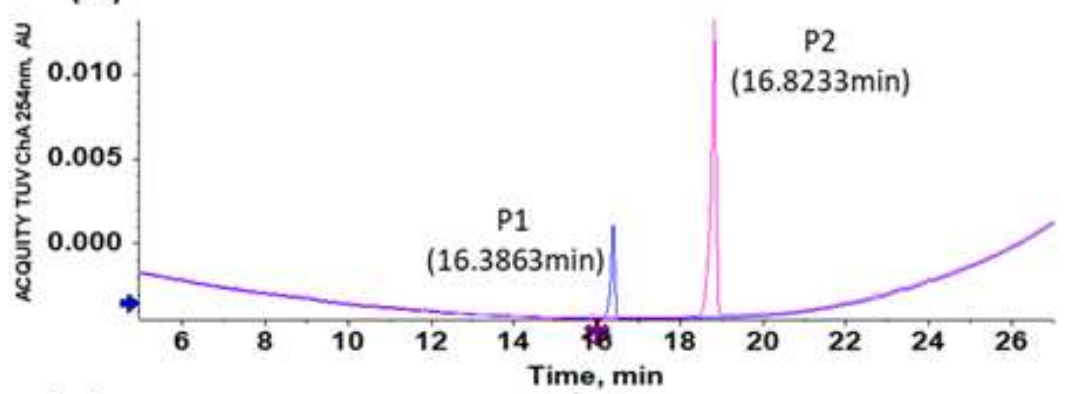

(B)

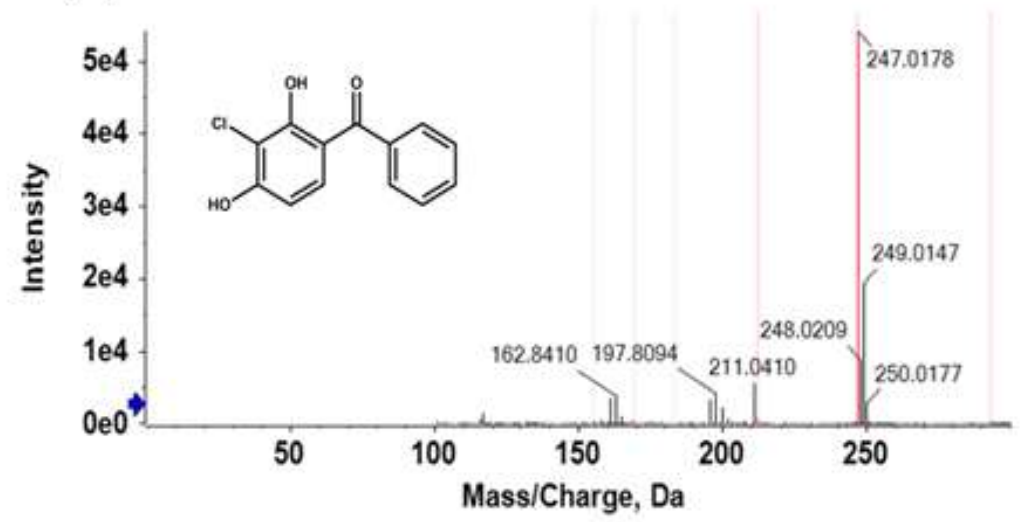

(C)

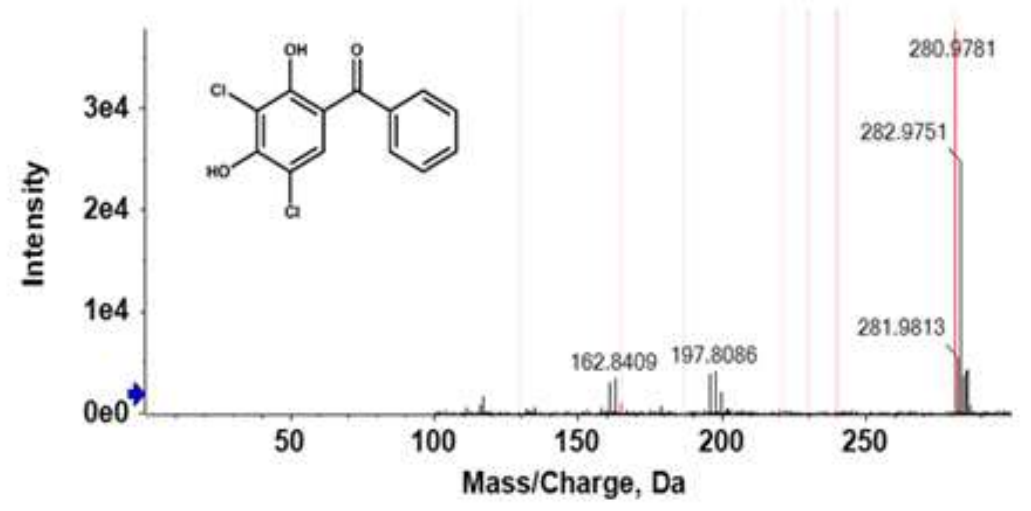

178

179 Figure $\mathbf{S 5}$ The chromatogram of P1 and P2 (A) separated from the reaction mixture,

180 the MS spectrum of separated P1 (B) and P2 (C).

181 


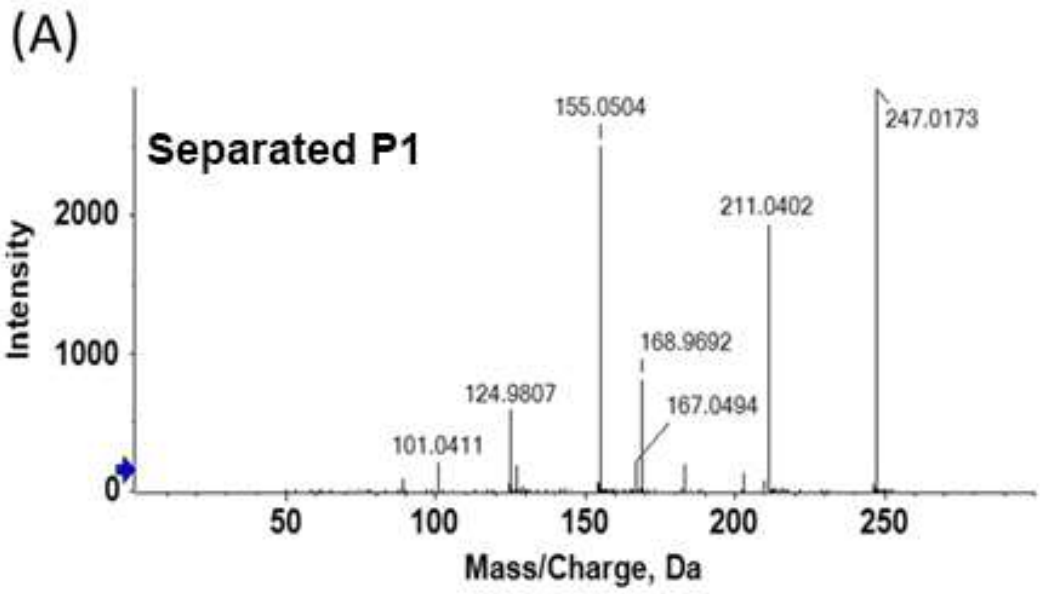

(B)

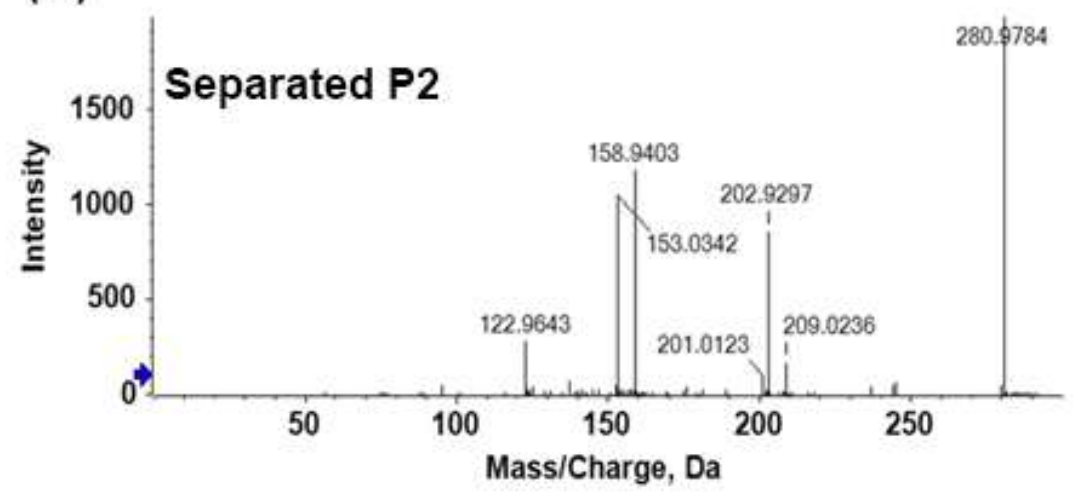

182

183 Figure S6 The MSMS spectrum of separated P1 (A) and P2 (B). 


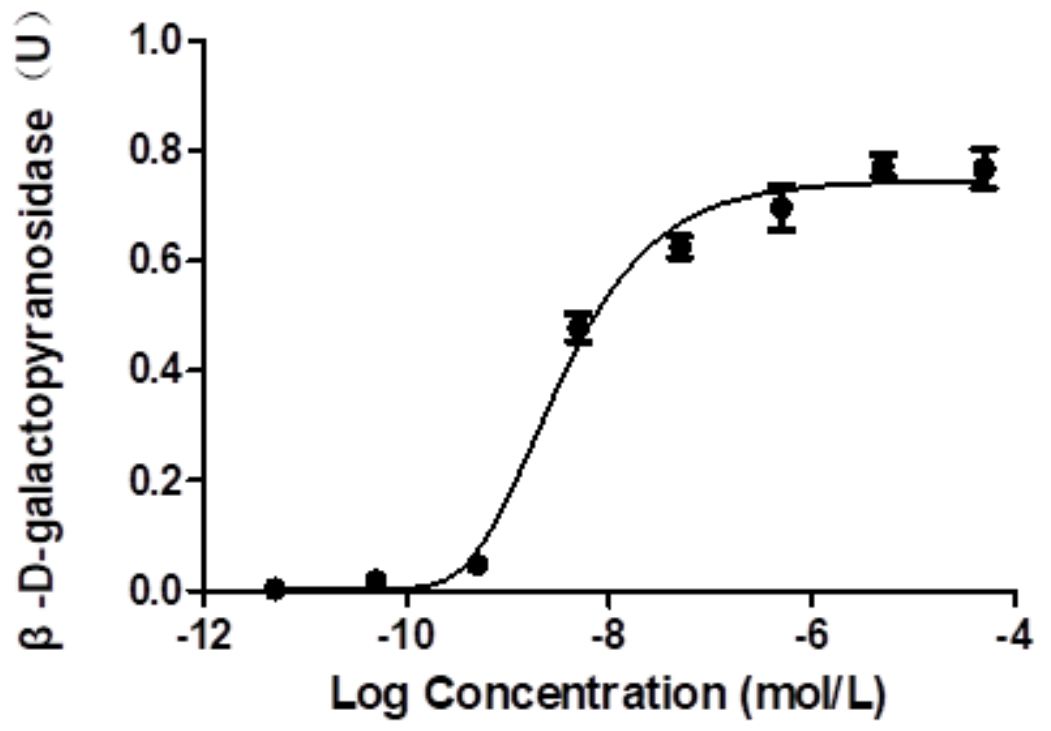

185

186 Figure S7 The standard dose-response curve for the positive control DHT by human

187 recombinant AR yeast two-hybrid assay. Error bars show the standard deviation of 188 triplicate assays. 


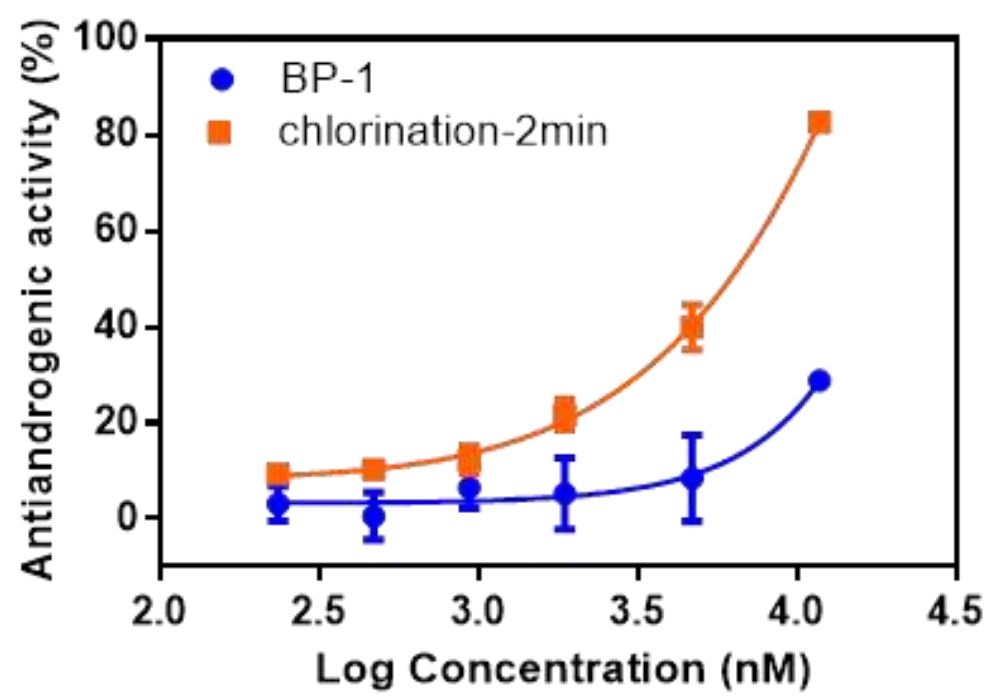

190

191 Figure S8 Antiandrogenic activity of BP-1 and its reaction mixture after $2 \mathrm{~min}$

192 chlorination using recombinant AR two-hybrid yeast assay; $\mathrm{pH}=7.0 ; 22 \pm 0.5^{\circ} \mathrm{C}$.

193 Values are presented as the mean \pm standard error of three biological triplicates. 


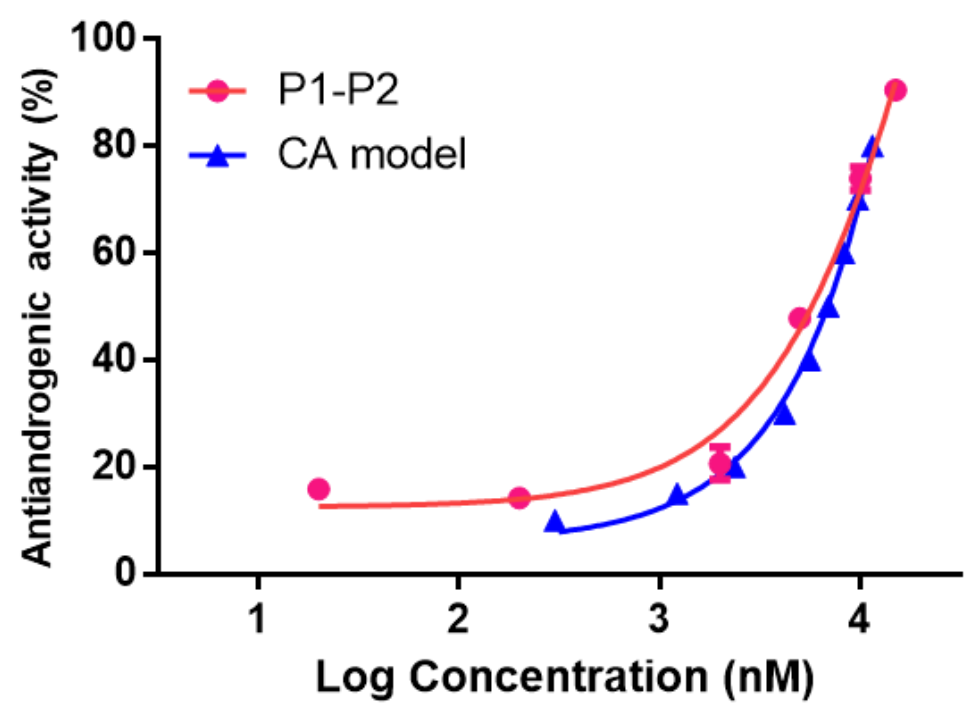

195

196 Figure S9 The observed (via recombinant AR two-hybrid yeast assay) and the

197 predicted (via CA model) AR antagonism effect of the mixture of P1-P2.

198 


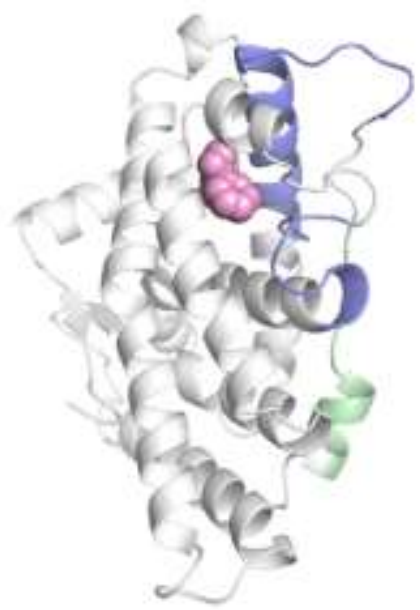

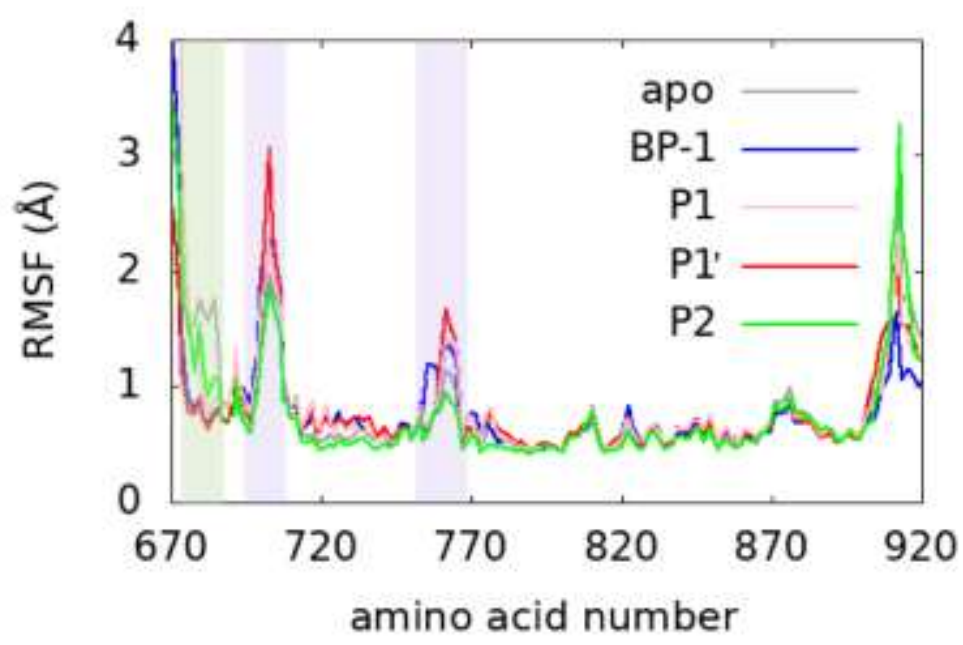

200 Figure S10 RMSF plot of ligand-free and ligand-bound AR LBD. The RMSF of the

201 C-alpha atoms of each amino acid was calculated according to 350-ns GaMD

202 trajectories. After ligand (pink) binding, part of the protein could become more

203 flexible (purple) or rigid (green). 


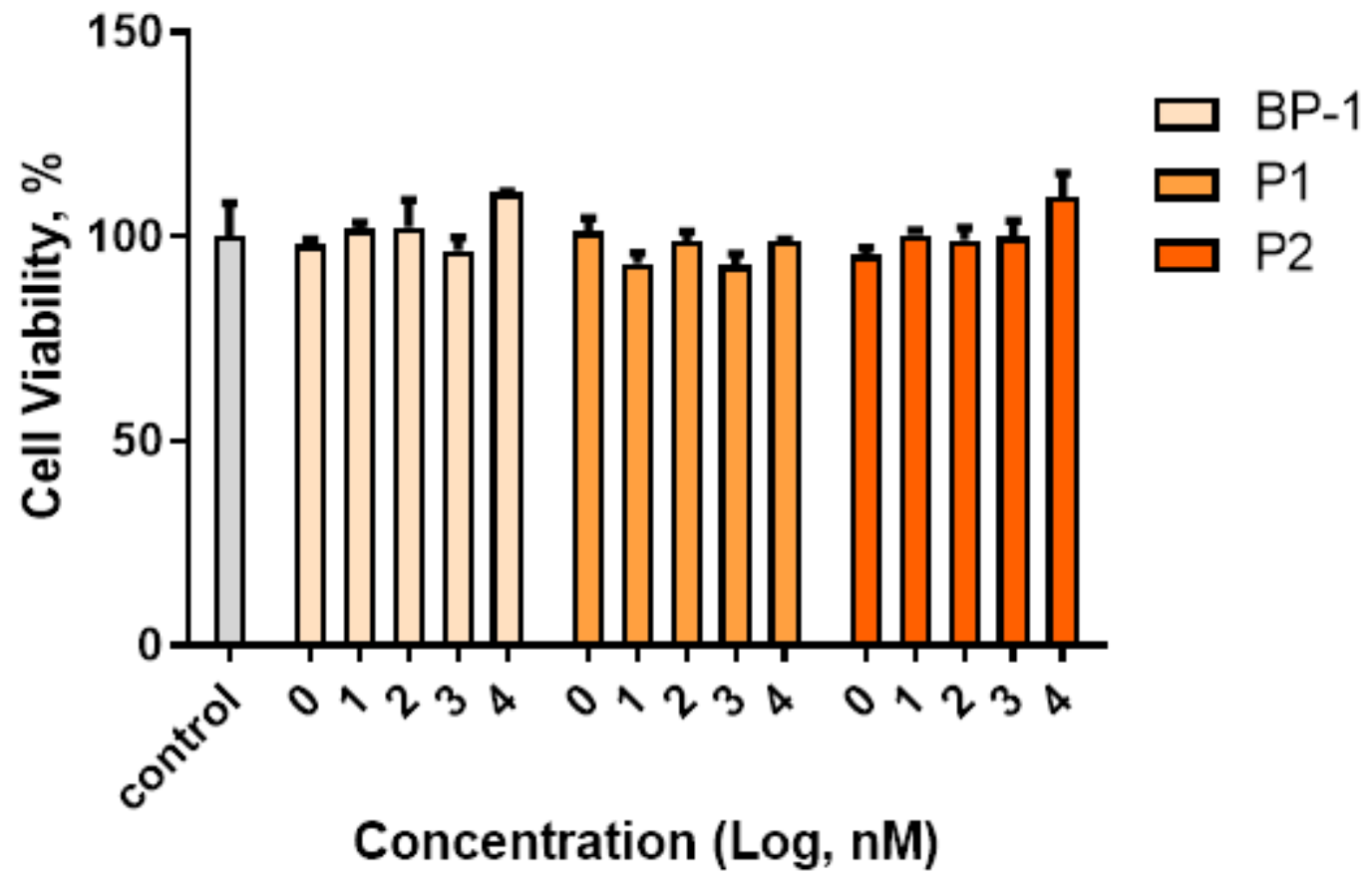

206 Figure S11 Cell viability upon exposure to BP-1, P1 and P2. LNCaP cells were

207 treated with BP-1, M1 and M2 with the concentrations ranging from $1 \mathrm{nM}$ to $10^{4} \mathrm{nM}$ 208 for $24 \mathrm{~h}$.

209 
211 1. Ding, K.; Kong, X.; Wang, J.; Lu, L.; Zhou, W.; Zhan, T.; Zhang, C.; Zhuang,

212 S., Side chains of parabens modulate antiandrogenic activity:in vitro and molecular 213 docking studies. Environ. Sci. Technol. 2017, 51, 6452-6460.

$214 \quad 2 . \quad$ Li, J.; Li, N.; Ma, M.; Giesy, J. P.; Wang, Z., In vitro profiling of the 215 endocrine disrupting potency of organochlorine pesticides. Toxicol. Lett. 2008, 183, $216 \quad 65-71$.

217 3. Jorgensen, W. L.; Chandrasekhar, J.; Madura, J. D.; Impey, R. W.; Klein, M. L., Comparison of simple potential functions for simulating liquid water. J. Chem. Phys. 1983, 79, 926-935.

$220 \quad 4 . \quad$ Friesner, R. A.; Banks, J. L.; Murphy, R. B.; Halgren, T. A.; Klicic, J. J.; Mainz, D. T.; Repasky, M. P.; Knoll, E. H.; Shelley, M.; Perry, J. K.; Shaw, D. E.; Francis, P.; Shenkin, P. S., Glide: A new approach for rapid, accurate docking and scoring. 1. Method and assessment of docking accuracy. J. Med. Chem. 2004, 47, 1739-1749. 5. Halgren, T. A.; Murphy, R. B.; Friesner, R. A.; Beard, H. S.; Frye, L. L.; Pollard, W. T.; Banks, J. L., Glide: A new approach for rapid, accurate docking and scoring. 2. Enrichment factors in database screening. J. Med. Chem. 2004, 47, 17501759.

229 6. Jorgensen, W. L.; Maxwell, D. S.; TiradoRives, J., Development and testing of 230 the OPLS all-atom force field on conformational energetics and properties of organic 231 liquids. J. Am. Chem. Soc. 1996, 118, 11225-11236.

$2327 . \quad$ Friesner, R. A.; Murphy, R. B.; Repasky, M. P.; Frye, L. L.; Greenwood, J. R.; Halgren, T. A.; Sanschagrin, P. C.; Mainz, D. T., Extra precision glide: Docking and scoring incorporating a model of hydrophobic enclosure for protein-ligand complexes. J. Med. Chem. 2006, 49, 6177-6196.

236 8. Roe, D. R.; Cheatham, T. E., PTRAJ and CPPTRAJ: Software for processing and analysis of molecular dynamics trajectory data. J. Chem. Theory Comput. 2013, 9, 238 3084-3095.

239 9. Humphrey, W.; Dalke, A.; Schulten, K., VMD: Visual molecular dynamics. $J$. 240 Mol. Graphics 1996, 14, 33-38.

241 10. Lange, O. F.; Grubmuller, H., Generalized correlation for biomolecular 242 dynamics. Proteins: Structure, Function, and Bioinformatics 2006, 62, 1053-1061.

243 11. Greenidge, P. A.; Kramer, C.; Mozziconacci, J.-C.; Wolf, R. M., MM/GBSA 244 binding energy prediction on the PDBbind data set: Successes, failures, and directions 245 for further improvement. J. Chem. Inf. Model. 2013, 53, 201-209.

246 12. Tan, C.; Tan, Y. H.; Luo, R., Implicit nonpolar solvent models. J. Phys. Chem. 247 B 2007, 111, 12263-12274.

248 13. Bashford, D.; Case, D. A., Generalized Born models of macromolecular 249 solvation effects. Annu. Rev. Phys. Chem. 2000, 51, 129-152.

250 14. Tanner, D. E.; Phillips, J. C.; Schulten, K., GPU/CPU algorithm for 251 Generalized Born/solvent-accessible surface area implicit solvent calculations. $J$. 252 Chem. Theory Comput. 2012, 8, 2521-2530. 